\title{
IonCCD Detector for Miniature Sector-Field Mass Spectrometer: Investigation of Peak Shape and Detector Surface Artifacts Induced by keV Ion Detection
}

\author{
Omar Hadjar, ${ }^{1}$ Thomas Schlathölter, ${ }^{2}$ Stephen Davila, ${ }^{3}$ Shane A. Catledge, ${ }^{4}$ Ken Kuhn, ${ }^{1}$ \\ Scott Kassan, ${ }^{1}$ Gottfried Kibelka, ${ }^{1}$ Chad Cameron, ${ }^{1}$ Guido F. Verbeck ${ }^{3}$ \\ ${ }^{1}$ OI Analytical/CMS Field Products, Pelham, AL, USA \\ ${ }^{2}$ KVI Atomic and Molecular Physics, University of Groningen, Groningen, The Netherlands \\ ${ }^{3}$ Department of Chemistry, University of North Texas, Denton, TX, USA \\ ${ }^{4}$ Department of Physics, Center for Nanoscale Materials and Biointegration (CNMB), University of Alabama at Birmingham, \\ Birmingham, AL, USA
}

\begin{abstract}
A recently described ion charge coupled device detector IonCCD (Sinha and Wadsworth, Rev. Sci. Instrum. 76(2), 2005; Hadjar, J. Am. Soc. Mass Spectrom. 22(4), 612-624, 2011) is implemented in a miniature mass spectrometer of sector-field instrument type and MattauchHerzog (MH)-geometry (Rev. Sci. Instrum. 62(11), 2618-2620, 1991; Burgoyne, Hieftje and Hites J. Am. Soc. Mass Spectrom. 8(4), 307-318, 1997; Nishiguchi, Eur. J. Mass Spectrom. 14 (1), 7-15, 2008) for simultaneous ion detection. In this article, we present first experimental evidence for the signature of energy loss the detected ion experiences in the detector material. The two energy loss processes involved at keV ion kinetic energies are electronic and nuclear stopping. Nuclear stopping is related to surface modification and thus damage of the lonCCD detector material. By application of the surface characterization techniques atomic force microscopy (AFM) and X-ray photoelectrons spectroscopy (XPS), we could show that the detector performance remains unaffected by ion impact for the parameter range observed in this study. Secondary electron emission from the (detector) surface is a feature typically related to electronic stopping. We show experimentally that the properties of the $\mathrm{MH}$-mass spectrometer used in the experiments, in combination with the IonCCD, are ideally suited for observation of these stopping related secondary electrons, which manifest in reproducible artifacts in the mass spectra. The magnitude of the artifacts is found to increase linearly as a function of detected ion velocity. The experimental findings are in agreement with detailed modeling of the ion trajectories in the mass spectrometer. By comparison of experiment and simulation, we show that a detector bias retarding the ions or an increase of the B-field of the IonCCD can efficiently suppress the artifact, which is necessary for quantitative mass spectrometry.
\end{abstract}

Electronic supplementary material The online version of this article (doi:10.1007/s13361-011-0213-x) contains supplementary material, which is available to authorized users.

Correspondence to: Omar Hadjar; e-mail: ohadjar@oico.com 
Key words: IonCCD, Charged particle array detector, Scan-free mass spectrometry, Sectorfield, Electronic and nuclear stopping power, Secondary electrons, keV ion-surface interaction, Surface characterization

$\mathrm{P}$ article-surface interactions are processes that form the very basis for a vast range of applied physics. Examples include particle detection, diagnostic surface characterization techniques such as secondary ion mass spectrometry (SIMS) [6-9], X-ray photon spectroscopy (XPS) [10], Auger electron spectroscopy (AES) [11, 12] and surface fabrication (lithography and chemical vapor deposition) [13-16]. When not directly exploited, particlesurface interactions often cause artifacts to be acknowledged, understood, and, if possible, eliminated. In particle detection applications, whether the particle is a photon or a particle with rest mass, most often detection is triggered by total or partial deposition of particle energy upon impact on the detector. Most popular ion detectors in the fields of physical chemistry, atomic and molecular physics that are also used in contemporary mass analyzers are channeltrons and microchannel plates (MCPs [17]), which are both based on secondary electron multiplication (SEM). As an initial step, these detectors rely on secondary electron emission induced by deposition of particle kinetic energy into the detector material. Subsequently, an avalanche of secondary electrons is generated due to an electric field along the detector channels. Ultimately, this leads to a detectable charge pulse, which can be used for ion counting, timing, position detection, and other purposes. Since secondary electron emission is the main aspect of this article, it is convenient to recapitulate some of its basic features. The yield of secondary electrons upon ion impact is proportional to the ion velocity [18] (kinetic electron emission, KEE) but also to the ion charge state [19] (potential electron emission, PEE). The influence of the latter is, for instance, due to (twoelectron) Auger processes. Due to efficient coupling to vibrational modes of the system, PEE is usually suppressed in multiply charged polyatomic systems [20, 21] most relevant in mass spectrometry. High accelerations $(>3 \mathrm{kV})$ leading to high kinetic energies are thus needed for optimum ion detection efficiencies. In the following, we will refer to such detectors as kinetic effect detectors, which inherently destroy the particle upon measurement. The complement are image charge detectors, used for instance in FT-ICR [22-24] and Orbitrap $[25,26]$ mass spectrometers usually associated with the highest resolving power $\left(R_{p}>100.000\right)$. Image charge detectors rely solely on the particle charge and can thus be classified as potential effect detectors. Detected ions are not lost and remain in the apparatus after detection.

A detector family that is rapidly gaining importance employs the emerging technology of pixilated array detectors such as Medipix/Timepix (developed by CERN and the Nikhef Institute in Amsterdam) for X-ray detection [27], velocity mapping imaging [28], and for mass spectrometric applications [29].
Other detection systems are used in the same field of 2D imaging with relatively high temporal resolution such as the NA64 Gigatracker pixel detector [30] and the PImMS sensor [31]. In the field of non-scanning sector-field mass spectrometry focal plane camera with CMOS technology [32] and IonCCD[1, 2] were successfully used. Those latter detectors are, rather, charge collectors with no temporal resolution capability as they do not provide single-particle detection capability. The IonCCD proved to be a practical imager for ion beam diagnostics at different vacuum stages in a custom built mass spectrometer [33]. This paper focuses on the IonCCD, employed here as a focal plane array for a MH-mass spectrometer. In their miniaturized form (ion path $<15 \mathrm{~cm}$ ), these instruments are best suited for low mass range applications $(<150 \mathrm{u})$. IonCCD operates in a charge integrating mode. Dispersed ions neutralize on the electrode pixels for a well-defined time, known as the integration time. While solely the charge of the incoming ions is used for detection, the kinetic effect can lead to artifacts. Naturally, such artifacts are strongest when relatively high particle velocities are involved. Sector-field instruments in general and MH-Mass spectrometer in particular call for high acceleration of relatively low mass ions for best performance. Accordingly, we observe a strong kinetic effect induced artifact on the IonCCD signal when coupled to a MH-Mass spectrometer: a very distinct negative peak located at the low mass side of the respective positive ion peak is observed, as reported in an earlier publication [32, 34].

It is the main purpose of this article to investigate the kinetic effect induced artifact thoroughly. After introduction the fundamentals of electronic stopping, we discuss the $3 \mathrm{D}$ modeling of ion trajectories, present experimental fingerprints of the artifact and propose solutions to eliminate the peak distortion. The suppression of the artifact would be very beneficial for quantitative mass spectrometry, especially for isotope ratio applications. In addition to electronic effects, ion surface interactions also feature processes summarized under the term nuclear stopping. Most relevant in this context are sputtering, implantation, and dislocation processes, since they can lead to degradation of the detector surface. We have used atomic force microscopy (AFM [35]) for surface topography studies and X-ray photoelectron spectroscopy (XPS [10]) to track chemical modification of the uppermost layers of the pixels.

\section{Stopping Power}

Ions have been used as probes for static and dynamic properties of matter already about one century ago [36-38] with electronic stopping in the $\mathrm{keV}$ region receiving particular interest from the 1980s until today [39-44]. 
Briefly, when a keV ion impinges into a medium, it experiences elastic energy loss (nuclear stopping) due to elastic nucleus-nucleus interaction and inelastic energy loss due to the friction forces experienced by the ion when passing through the valence electron distribution of the medium. While the nuclear stopping dominates at lower particle velocity $(\mathrm{v}<0.01 \mathrm{a}$.u. $)$ and is proportional to $1 / \mathrm{v}$, the inelastic energy loss (or electronic stopping) dominates at high velocities ( $>>0.1$ a.u.). For $\mathrm{keV}$ ion energies and below the Fermi velocity, electronic stopping is caused by inelastic scattering of valence electrons from the screened potential of a moving ion. Each inelastic scattering event leads to generation of an electron-hole pair [45] prevails and the deposited energy can be approximated as directly proportional to projectile velocity [46] when approximating the valence electrons as a free electron gas:

$$
S=\frac{d E_{k}}{d X}=v \times \gamma\left(r_{s}, Z\right) ; \text { with } r_{s}=\left(\frac{4}{3} \pi n_{e}\right)^{-\frac{1}{3}}
$$

Here $E_{k}$ is the particle kinetic energy and $\mathrm{X}$ is the distance traveled in the medium. Through $r_{s}$ the friction coefficient $\gamma$ is a function of the electron density $n_{e}$ of the medium and of the ion nuclear charge $\mathrm{Z}$. In quantum scattering with partial wave representation, the partial scattering amplitude can be expressed as the phase shift [47], from which $\gamma$ can be calculated [40]. The linearity of Equation (1) was not only demonstrated for solid state targets but also for gas phase cluster targets [48, 49]. For a given electron density, $\gamma$ exhibits an oscillatory behavior with Z [44], which was experimentally observed as well [50-53]. Minimum stopping power is observed for closed shell (rare gas) configurations, while maximum stopping power is given by open shell configurations of ions embedded in an electron gas of given density.

How does electronic stopping translate into secondary electron emission? When occurring close to a surface, electron hole pair creation passes into direct electron emission. Furthermore, electronic excitation can couple to nuclear degrees of freedom, resulting in structural changes (sputtering, fragmentation) and local heating, which can lead to thermionic electron emission. The number of electrons produced during an ion-matter interaction process is defined as secondary electron yield (SEY), which is the very process SEM type detectors are based upon. A systematic study on the effect of different surfaces on SEY can be found in [54]. Besides ion velocity $v$ and atomic number $Z$, the ion charge state $q$ can affect the SEY through the potential effect which is typically negligible for polyatomic ions $[50,55,56]$. Note that ion charge is the very parameter charge sensitive detectors such as image charge detectors, Faraday cup collectors and IonCCDs are based upon.

For ions in the kinetic energy range under study here, the energy distribution of the secondary electrons peaks between a few $\mathrm{eV}$ and the $10 \mathrm{eV}$ range. About one electron per incoming ion is emitted at a kinetic energy of $1 \mathrm{keV} / \mathrm{u}-\mathrm{a}$ yield that typically increases linear with ion velocity for a given ion-surface system [57].

\section{Experimental}

All data presented in this work are produced using a miniature MH-mass spectrometer [3, 58], which is widely used in compact transportable MS and GC/MS products (IonCam). The instrument is shown in Figure 1 and its parameters are summarized in Table 1. An electron impact (EI) ion source is used and typically operated at 70-eV electron energy. Here, He,

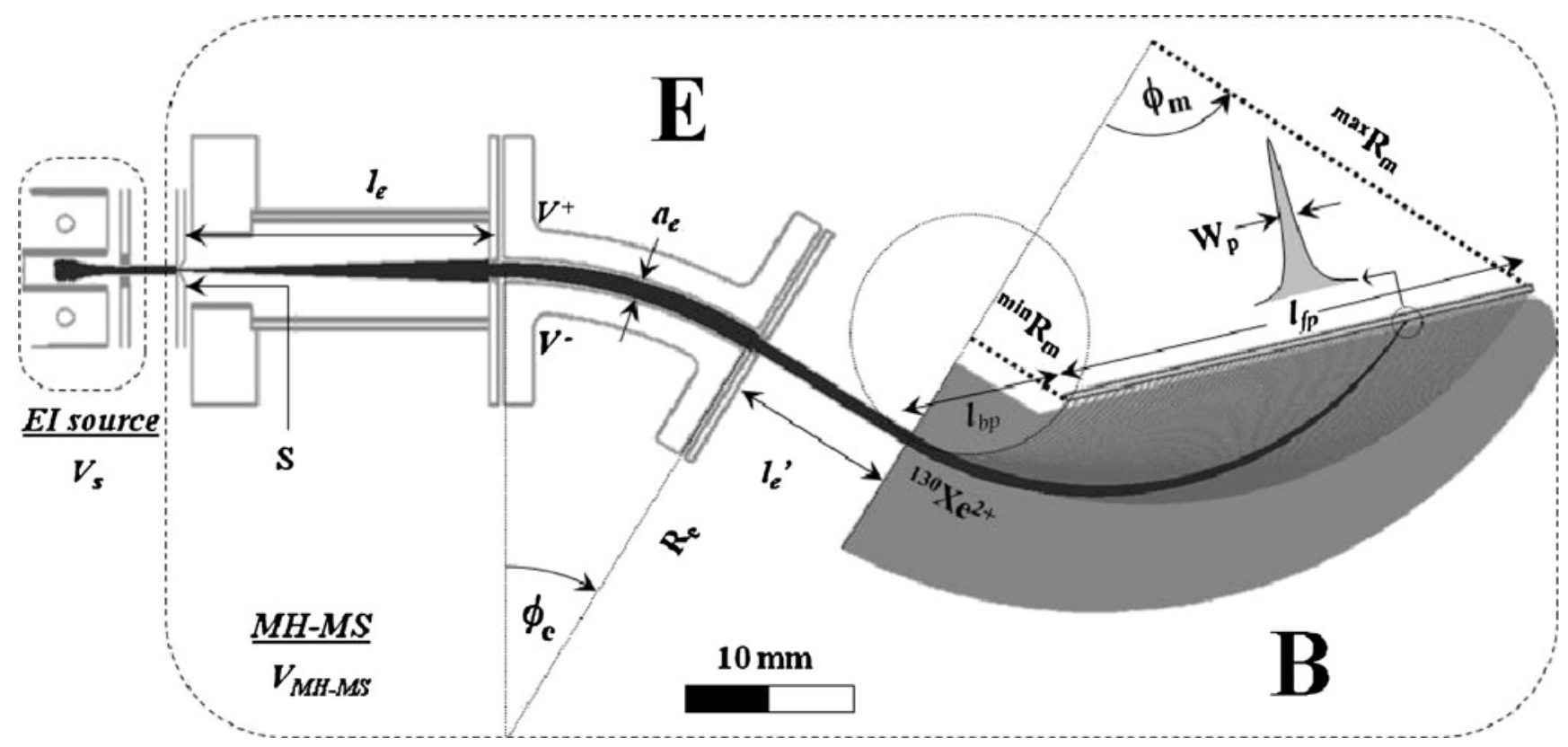

Figure 1. Schematic of the used miniature MH-mass spectrometer coupled to an El source. ${ }^{130} \mathrm{Xe}^{2+}$ ion trajectories are shown together with a single trajectory per $\mathrm{m} / \mathrm{z}$ spanning the whole mass window to illustrate the instrument's operation principle 
Table 1. Parameters Describing the Miniature MH-mass Spectrometer and its Performance

\begin{tabular}{|c|c|c|}
\hline Parameter & Description & Value \\
\hline$V_{S}$ & EI source bias & {$[+250,+2000] \mathrm{V}$} \\
\hline$V_{\mathrm{MH}-m a s s}$ spectrometer & Analyzer bias & $0 \mathrm{~V}$ \\
\hline$V_{I o n C C D}$ & IonCCD floating voltage & {$[-3000,+3000] \mathrm{V}$} \\
\hline$S$ & Object slit width & $100 \mu \mathrm{m}$ \\
\hline$l_{e}=R_{e} / \sqrt{ } 2$ & Oject slit-to-ESA entrance distance & $35.4 \mathrm{~mm}$ \\
\hline$\Phi_{e}$ & Electrostatic deflection angle & $31.8^{\mathrm{o}}$ \\
\hline$R_{e}$ & ESA radius & $50 \mathrm{~mm}$ \\
\hline$a_{e}$ & ESA electrodes gap & $2.5 \mathrm{~mm}$ \\
\hline$E=\left(V^{+}-V\right) / a_{e}$ & Electric field & $3.6^{*}\left(\mathrm{~V}_{\mathrm{S}}-\mathrm{V}_{\mathrm{MH} \text {-mass spectrometer }}\right) \mathrm{V} / \mathrm{cm}$ \\
\hline$l_{e}{ }^{\prime}$ & ESA exit-to-magnet entrance distance & $10 \mathrm{~mm}$ \\
\hline$l_{b p}$ & Blind plane length: magnet entrance-to-first pixel distance & $17 \mathrm{~mm}$ \\
\hline$\Phi_{m}$ & Magnetic deflection angle & $90^{\circ}$ \\
\hline$R_{m}^{m}$ & Ion magnetic trajectory radius & {$[12.7,48.8] \mathrm{mm}$} \\
\hline$a_{m}$ & Magnetic poles gap & $1.5 \mathrm{~mm}$ \\
\hline$B$ & Magnetic field & $1 \mathrm{~T}$ \\
\hline$l_{m}{ }^{\prime}$ & Magnet exit-to-IonCCD distance & $0.7 \mathrm{~mm}$ \\
\hline$B^{\prime}$ & Magnetic field at the IonCCD & $0.2 \mathrm{~T}$ \\
\hline$l_{f p}$ & Focal plane length & $51.0 \mathrm{~mm}$ \\
\hline$m_{\max } / m_{\min }$ & Dynamic mass range (see text) & 16 \\
\hline$R_{p}=R_{e} / 4 S$ & Resolving power at $5 \%$ Gaussian peak height ( $10 \%$ valley) & 125 \\
\hline $\operatorname{Tr}$ & Total transmission & {$[0.1,1] \%$} \\
\hline
\end{tabular}

$\mathrm{N}_{2}$, and Ar were introduced into the ion source from a reservoir at atmospheric pressure connected to the source through a $40 \mathrm{~cm}$ long capillary of $50 \mu \mathrm{m}$ diameter, serving as a flow limiter. During $\mathrm{N}_{2}$-operation of the ion source, the background pressure in the analyzer increased to about $10^{-5}$ torr. The pressure was monitored by a full range gauge (MKS-972B), containing a pirani module for low vacuum operation and a cold cathode module which automatically activates at about 5 $10^{-4}$ torr and operates down to $10^{-8}$ torr.

The MH-mass spectrometer employs a combination of a radial electric field and a homogeneous magnetic field in order to obtain velocity focusing and spatial focusing over the entire focal plane simultaneously and for all masses. We use a sintered permanent magnet of Neodymium-Iron-Boron $(\mathrm{NdFeB})$ type located in vacuum. Nickel coating of the magnet prevents oxidation. The magnet assembly gives rise to a quasi-homogeneous $1 \mathrm{~T}$ B-field over the whole $1.5 \mathrm{~mm}$ gap between the poles, serving as ion flight-path region (see Figure S1 in Supplemental Information). For experiments using $\mathrm{He}^{+}$or protons, the magnet was demagnetized down to $0.38 \mathrm{~T}$. This allowed for low mass detection down to $1 \mathrm{kV}$ acceleration voltage. For the experiments presented here, the MH-mass spectrometer system was grounded and the ion acceleration voltage was defined by the floating bias of the EI source ( 250 and $2000 \mathrm{~V}$ ). Operation of the IonCCD at ground potential ensures a field free region at interface between magnet exit and IonCCD. In order to float the IonCCD, digital processing board, and fiber optic data links are enclosed in a Plexiglas enclosure for safety and are electrically isolated from ground through an AC line isolation transformer (Triad VPS-230-350) connected to the respective power supplies. The fiber optic data links consists of a pair of Ethernet transceivers (S-110-M2ST2) and a pair of controller-area network CAN-bus transceivers (LUXLink DX-7601-1) with two 6-foot long ST type connector dual fiber optic cables providing high voltage isolation for both the Ethernet and CANbus data communications between the digital processing board and a laptop computer. A save high voltage (SHV) connector on the enclosure connects to an external high voltage power supply to bias the IonCCD and the camera electronics to the desired high voltage. The IonCCD bias can be used to ensure the field free region condition to be maintained independent of other bias voltages. Grounded sources could for instance be coupled to an MH-mass spectrometer equipped with IonCCD, but also other applications such as mesh-free beam profiler under high voltage conditions are possible. On the other hand a controllable potential gradient in the region between IonCCD and magnet exit face can be used either to retard or accelerate the secondary electrons from the IonCCD surface (this feature will be exploited to investigate the respective artifact in the mass spectra).

Now, ion dispersion in the magnetic field obeys the equation:

$$
R_{m}=\frac{1}{B} \sqrt{2 \times \frac{m}{q} \times\left(V_{s}-V_{M H-M S}\right)}
$$

with $\mathrm{m}$ and $\mathrm{q}$ being the mass and charge of the ion, $\mathrm{V}_{\mathrm{S}}$ the bias voltage of the EI source bias voltage, $\mathrm{V}_{\mathrm{MH} \text {-mass spectrometer the }}$ $\mathrm{MH}$-mass spectrometer analyzer bias voltage and $\mathrm{B}$ the magnitude of the B-field.

The IonCCD used as a focal plane array detector was described recently, elsewhere [2]. Briefly it consists of a $2126 \times$ 1 pixel array spanning $51-\mathrm{mm}$ with $21 \times 1500 \mu \mathrm{m}^{2}$ pixel area. The length of the array matches the magnet exit face that determines the focal plane with the pixel height being identical to the width of the magnetic sector ga. The pixel top most layer is $200-\mathrm{nm}$ TiN on top of $100-\mathrm{nm} \mathrm{Al}$ with a $\mathrm{SiO}_{2}$ substrate. Note that the TiN layer probably leads to a lowered SEY as compared to the Al substrate - an effect which has been observed for TiNcoated alumina ceramics in RF windows [59].

The pixels are separated by a $3-\mu \mathrm{m} \mathrm{SiO}_{2}$ insulating gap providing a $24-\mu \mathrm{m}$ pitch and an effective detection area or a pixel area ratio (PAR) of $88 \%$. The IonCCD accumulates 
the charge of the incoming charged particle for a welldefined integration time between $83 \mu$ s and $5 \mathrm{~s}$. The total charge gives rise to a voltage due to the capacitive nature of the pixels. As a last step, the 2126 individual voltage values are serially read during the $2.7 \mathrm{~ms}$ readout time and digitized by a 16-bit analogue-to-digital converter (ADC). The resulting spectrum of pixel number versus voltage is stored. The linear dynamic range exceeds $10^{3}$ with a noise floor of 9-dN for integration times less than $100 \mathrm{~ms}$. The focused $\mathrm{m} / \mathrm{z}$ ions produce a quasi-Gaussian distribution on the IonCCD (see Figure 1) with a FWHM given by: $W_{p}=\sqrt{2} \times S \times R_{m} / R_{e}$, where $\mathrm{S}$ is the analyzer opening slit width and $\mathrm{R}_{\mathrm{e}}$ is the electrostatic radius. While the peak width increases with $\mathrm{m} / \mathrm{z}$, the resolving power $R_{p}$ of the system is constant over the whole mass range under study: $R_{p}=R_{e} / 4 S$. Note that the mass range defined as the ratio between highest and lowest mass that still pass the analyzer is constant and depends solely on the analyzer geometry as follows:

$$
m_{\max } / m_{\min }=\left(1+l_{f p} / l_{b p}\right)^{2}
$$

Here $l_{f p}$ is the focal plane length matching the IonCCD array and the blind plane length $l_{b p}$ is the length between the magnet entrance face and the impact site of the ion with the lowest detectable mass (see Figure 1). $l_{b p}$ defines the minimum curvature radius of the respective ion. For the MH-mass spectrometer used in this work, we find $m_{\max } / m_{\min }=16$ (for instance allowing simultaneous detection $\mathrm{H}^{+}$up to ${ }^{16} \mathrm{O}^{+}$). From Equation (3) it is obvious that $m_{\max } / m_{\min }$ can be increased by increasing $l_{f p}$ or by decreasing $l_{b}$ An increase in $l_{f p}$ requiring a longer IonCCD array and a systematic increase in mass spectrometer size is expensive. However, $l_{b p}$ can be easily decreased by translating the IonCCD towards the magnet entrance. A prototype high dynamic mass range (HDMR) magnet assembly with $l_{b p}$ reduced from $17 \mathrm{~mm}$ to $6 \mathrm{~mm}$ has already been constructed and we found $m_{\max } / m_{\min }$ to exceed 72 (e.g., $\mathrm{H}^{+}$up to $\mathrm{C}_{5} \mathrm{H}_{12}{ }^{+}$).

Furthermore, the mass range of the instrument depends on ion acceleration, geometry and B-field of the instrument as follows:

$$
m_{\max }-m_{\min }=\left(\frac{q B^{2}}{4 V_{s}}\right) \times l_{f p} \times\left(l_{f p}+l_{b p}\right)
$$

For correct simulations of ion trajectories in front of the detector surface, the magnetic field needs to be mapped in the respective region. We determined $\mathrm{B}=0.2 \mathrm{~T}$ at the detector surface located $0.7 \mathrm{~mm}$ from the magnet exit face, $\mathrm{B}=0.4 \mathrm{~T}$ at the magnet exit face, and $1 \mathrm{~T} 5 \mathrm{~mm}$ inside the magnet.

In order to study the nuclear stopping effect of $\mathrm{keV}$ ion impact upon the IonCCD surface we employed two surface characterization techniques; Atomic Force Microscopy (AFM) and X-ray photon spectroscopy (XPS).
AFM was performed using a Veeco Explorer operating in contact mode with a non-conductive silicon nitride tip having nominal tip radius of $20 \mathrm{~nm}$. XPS was performed using a PHI VersaProbe Scanning XPS Microprobe (Physical Electronics, Chanhassen, MN, USA) equipped with a high flux aluminum anode X-ray source that provides a highly focused monochromatic X-ray beam. A $100 \mu \mathrm{m}$ X-ray spot size allows for the analysis of a large area of the exposed pixels' area. The IonCCD was scanned at areas where the pixels had been exposed to the $\mathrm{N}_{2}{ }^{+}$ions and at areas where the pixels have had no exposure to $\mathrm{N}_{2}^{+}$ions. The same study was performed for $\mathrm{Xe}^{+}$exposed areas.

\section{Simulation}

Ion trajectories in the combined electric and magnetic fields of the entire MH-mass spectrometer instrument were computed using the SIMION 8.0 package in 3D mode. For the simulations we have chosen four types of singly charged ions with masses covering most of the mass window at a given acceleration voltage and/or B-field. Each data point in Figure 2 is the result of 20,000 trajectories, each originating from a cylindrical ionization volume of $2 \mathrm{~mm}$ length and $1 \mathrm{~mm}$ radius. The 3D initial momenta of the ions were isotropically distributed over the full angular range with velocities corresponding to a room temperature thermal energy of $36 \mathrm{meV}$. All relevant properties of the system Equations (2), (3) and (4) are summarized in Figure 2. Figure 2a displays the spatial peak width at half maximum as a function of the detected ion mass. Here, the ion impact site on the IonCCD was recorded in 2D, from which a 1D histogram was constructed to mimic the IonCCD signal. The $\mathrm{Y}$ impact coordinates (along the B-field) were integrated while the $\mathrm{X}$ coordinates (along the focal plane) were sorted into $24 \mu \mathrm{m}$ wide bins corresponding to the experimental detector pitch. The simulation produces focused $\mathrm{m} / \mathrm{z}$ beams with Gaussian peak shapes similar to the experimentally observed peaks as shown in Figure S2 (in Supplemental Information). Figure $2 \mathrm{~b}$ shows the resolving power of the system, which is almost constant around a value of 125 calculated at $5 \%$ peak height. This percentage value is selected since it leads to a $10 \%$ valley between adjacent peaks with equal height according to the definition of resolving power in sector-field instruments. Note that Figure $2 \mathrm{~b}$ shows a systematic lower resolving power for low mass. This is due to the fact that the focusing of high curvature trajectories is very sensitive to $l_{m}$ ' (see Table 1). Figure 2c nicely shows the increase of the mass resolution with the mass of the ion to be resolved, which is a direct effect of the system's constant resolving power. From the 3D simulation we can also extract the instrument total transmission, defined as the ratio of the number of ions detected by the IonCCD and ions formed in the ionization volume. The results are shown in Figure 2d. Clearly the instrument transmission not only increases linearly with the 

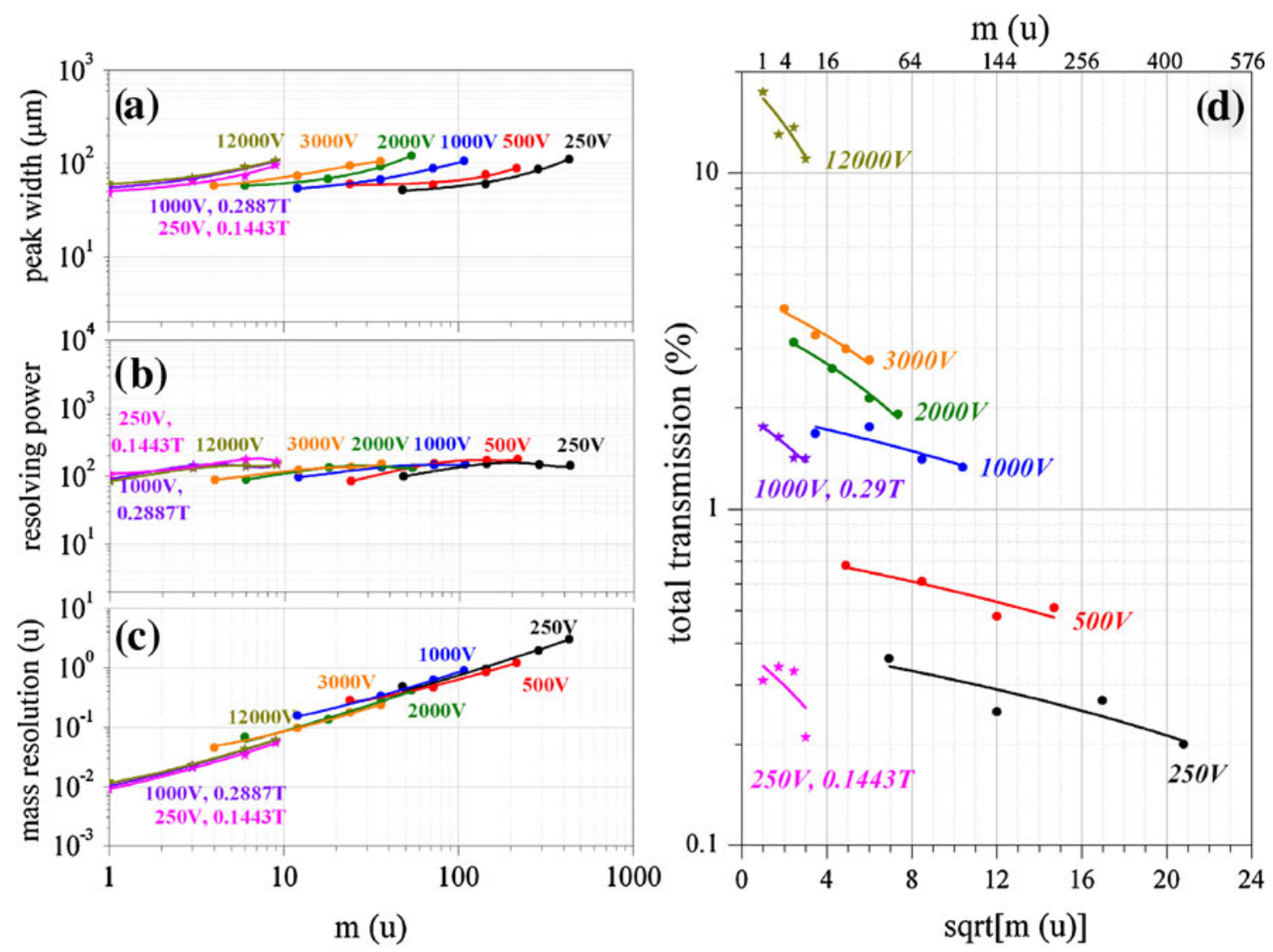

Figure 2. Results extracted from a full 3D simulation of the instrument from the ionization volume to the detection area. (a) Peak width as function the ion mass. (b) Resolving power of the instrument based on $5 \%$ peak height. (c) Mass resolution at $5 \%$ peak height. (d) Overall instrument transmission as function of the acceleration voltage

acceleration voltage, but for constant acceleration decreases linearly with the ion magnetic radius or $\sqrt{m}$. The transmission curve is a necessary input for the correction of the recorded intensities. We also simulated different B-fields and the corresponding accelerations in order to obtain the same detection mass window. Masses 1, 3, 6, and 9 u were simulated such that they fall onto the same pixels (identical magnetic radii for the different settings). This allows us to decouple the magnetic sector transmission function from the electrostatic part of the instrument. The following settings were used: $(12000 \mathrm{~V}, 1 \mathrm{~T}),(1000 \mathrm{~V}, 0.29 \mathrm{~T})$, and $(250 \mathrm{~V}$, $0.14 \mathrm{~T}$ ). As shown in Figure 2, those settings lead to identical resolution but different transmissions, with transmission increasing linearly with acceleration field. The latter is due to the fact that the instrument has no transversal confinement capabilities. Note that the presented transmissions are highly dependent on the starting volume, which is inversely proportional to the overall transmission. We have also studied the influence of a magnetic EI source on the performance of the system. Experiment and simulation show a dramatic increase of the total system transmission by about one order of magnitude. This is due to a better confinement of the ionizing electron beam which reduces the ionization volume.

\section{Results and Discussion}

\section{Simulation}

SIMION 3D-trajectory simulations were used to determine the area on the detector surface that is exposed to initially detected ions for a given set of parameters. From here, secondary electrons are emitted which eventually return back to the detector.

The following three particle distributions then determine the actual signal on the IonCCD:

i) $D_{1}$ is the incoming ion distribution as obtained from the SIMION simulation of the full instrument. $\mathrm{D}_{1}$ is considered a positive contribution as the detected positive ions lead to a positive signal on the IonCCD output with a distribution width defined by the instrument parameters. The absolute amplitude of $\mathrm{D}_{1}$ depends on the instrument transmission.

ii) $\mathrm{D}_{2}$ is the distribution of the outgoing or emitted secondary electrons originating from $\mathrm{D}_{1}$ and weighted by the relative electron yield $\gamma$. For instance if $0.1 \mathrm{e} / \mathrm{ion}$ is used as the electron yield, $D_{2}$ would be $D_{1} / 10$. $D_{2}$ has a positive sign since (positive) holes remain at the detector surface. 
iii) $\mathrm{D}_{3}$ is the incoming electron distribution due to $\mathrm{D}_{2}$ secondary electrons returning to the IonCCD surface under the influence of the B-field. $\mathrm{D}_{3}$ is considered as a negative contribution since it involves negative charges impinging on the surface. As sketched in S.I.-Figure 1, $\mathrm{D}_{3}$ is displaced with respect to $\mathrm{D}_{2}$ along the focal plane towards the low ion mass side. The distance of the centroids of $D_{2}$ and $D_{3}$ is:

$$
d_{e}=2 \times R_{m}^{e}=\frac{2}{q_{e} B^{\prime}} \times \sqrt{2 \times m_{e} \times E_{k}^{e}}
$$

Here $R_{m}^{e}$ is the electron curvature radius; $m_{e}$ and $q_{e}$ are electron mass and charge. $\mathrm{B}$ ' is the B-field on the IonCCD ranging from $0.2-\mathrm{T}$ at the surface to $0.25-\mathrm{T}$ at $\mathrm{R}_{\mathrm{m}}^{\mathrm{e}}=30 \mu \mathrm{m}$ (see Figure $\mathrm{S} 2$ in Supplemental Information); $\mathrm{E}_{\mathrm{k}}^{\mathrm{e}}$ is the electron kinetic energy, which was chosen as a Gaussian distribution centered around $6 \mathrm{eV}$ with $3 \mathrm{eV}$ FWHM. The solid angle of electron emission was chosen to be $30^{\circ}$ FWHM along $\mathrm{X}$ and $\mathrm{Y}$-axis of the detector array for the simulation of $\mathrm{D}_{3}$ distribution. $\mathrm{D}_{3}$ is dependent on the electron energy distribution, the magnetic field on the surface vicinity and finally the potential gradient in the interface magnetic exit face-IonCCD. The latter two effects are obvious from Figure S2 in Supplemental Information. which displays the simulated displaced secondary electron distributions for constant detector bias at different B-field magnitudes and vice versa. Coordinates are relative to the $D_{2}$ centroid, i.e. for given bias and B-field $d_{e}$ is the peak position of the respective electron distribution. For $\mathrm{B}=$ $0.2 \mathrm{~T}$ (experimentally measured), $\mathrm{d}_{\mathrm{e}}=60-\mu \mathrm{m}$ is obtained from the simulation shown in Figure S2a in Supplemental Information.

At sufficiently high B-field, i.e., for situations where the IonCCD is closer to the magnet exit face, or for higher retarding fields, $d_{e}$ becomes negligible with respect to pixel dimension. $\mathrm{D}_{3}$ then overlaps with $\mathrm{D}_{2}$ and both contributions cancel, leaving $\mathrm{D}_{1}$ the only contribution to the detected signal so that no effects of secondary electrons due to electronic stopping are observable.

Figure 3 shows the sum of the three distributions, i.e. the simulated total detector signal for zero detector bias at different $\mathrm{B}$ (a) and for $\mathrm{B}=0.2 \mathrm{~T}$ at different bias voltages. Both low B-field and zero retarding field lead to large
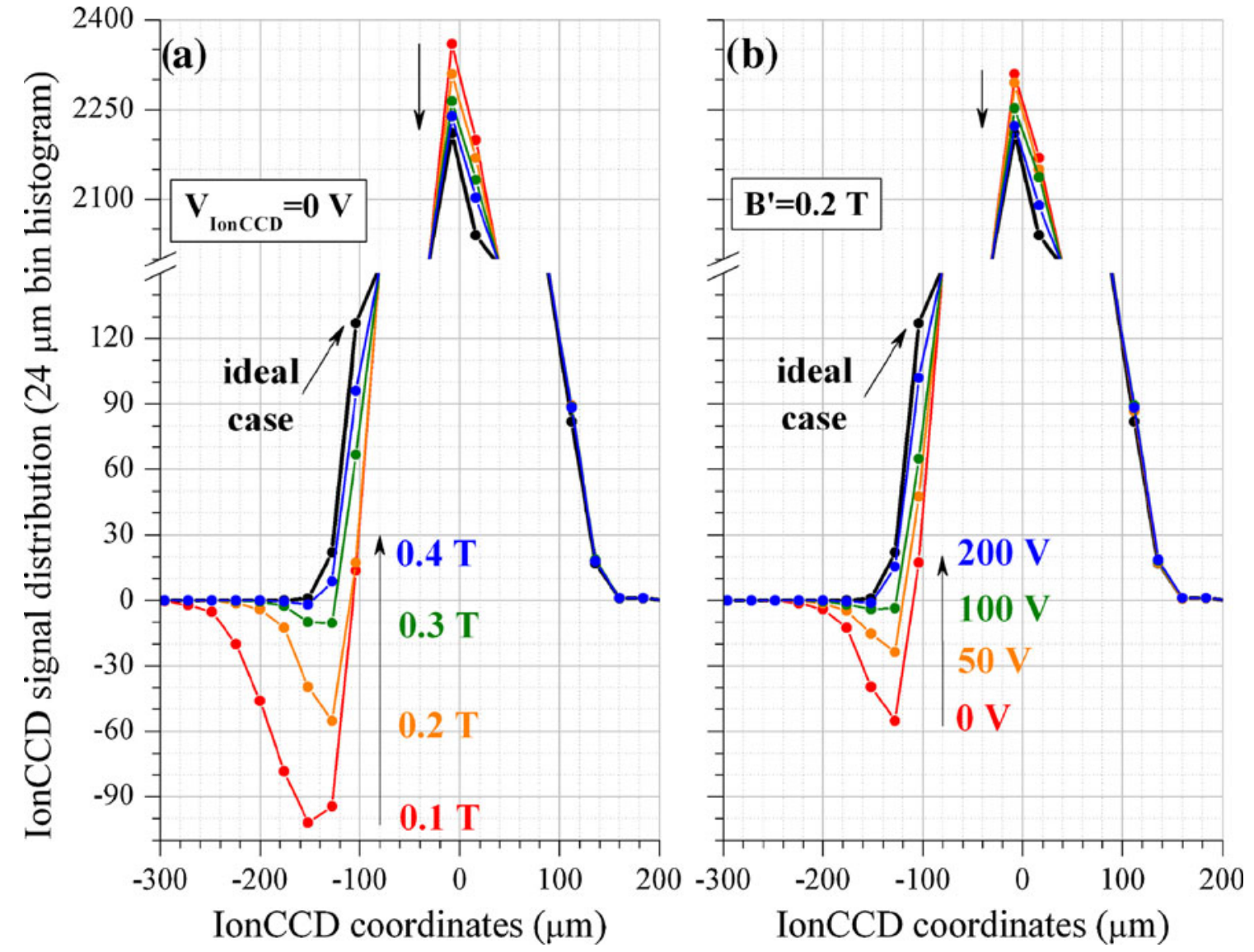

Figure 3. Simulated lonCCD signal expressed as the sum of the three simulated distributions: incoming ions, outgoing electrons and incoming electrons. The break in Y-axis is used to show the low signal artifact. (a) B-field effect on the negative peak. (b) Magnet-IonCCD retarding field effect on the negative peak 
displacement of $\mathrm{D}_{3}$. The total signal exhibits a clear and relatively deep depression on the low mass side and a small increase of the major peak. Larger B-field magnitudes of larger detector bias reduce the displacement and suppress the secondary electron induced artifact. This is especially important in isotope ratio mass spectrometry (IRMS).

Eventually, we also varied the centroid of the secondary electron kinetic energy over the range from 1 to $10 \mathrm{eV}$ and observed only negligible influences on the total detected signal. The same was found for variation of the secondary electron solid angle of emission.

For an easy comparison of simulated results with the experimental observations it is convenient to define a quantity characteristic for the secondary electron related artifact: we choose the ratio between integrated negative detector signal and the integrated positive signal $\mathrm{R}_{\mathrm{n} / \text {. For the }}$ simulated data, this ratio can be easily extracted from the distributions presented in Figure 3. Figure S3 in Supplemental Information shows $R_{n / p}$ as a function of the $B$-field on the IonCCD (B'), the FWHM of the peak on the detector $\left(\mathrm{W}_{\mathrm{p}}\right)$, the detector bias $\left(\mathrm{V}_{\text {IonCCD }}\right)$ and the electron yield $(\gamma)$. The reference setting is $\mathrm{B}^{\prime}=0.2 \mathrm{~T}, \mathrm{~W}_{\mathrm{p}}=100 \mu \mathrm{m}, \mathrm{V}_{\text {IonCCD }}=0 \mathrm{~V}, \gamma=0.1$. The first three parameters are either experimental settings ( $\mathrm{B}^{\prime}$ and $\left.\mathrm{V}_{\text {IonCCD }}\right)$ or observables $\left(\mathrm{W}_{\mathrm{p}}\right)$, whereas $\gamma$ is a relatively free parameter, depending on the TiN detector surface material, ion energy and ion species. Clearly, $\mathrm{R}_{\mathrm{n}}$ $p$ depends strongly on all four parameters, rendering the quantification of the electronic stopping related contribution more difficult. The implications of Figure S3 in Supplemental Information. are thus of crucial importance as they show that for a given ion-surface combination, $\mathrm{R}_{\mathrm{n}}$ $\mathrm{p}$ will be different in different instruments, as relevant parameters vary.

However, for a given ion-surface system and identical instrument design the artifact is reproducible. From Figure S3 in Supplemental Information, it is clear that if the IonCCD is placed right at the magnet exit (operation at $0.4-\mathrm{T}$ ) $\mathrm{R}_{\mathrm{n} / \mathrm{p}}$ is $<10^{-2}$ $\%$. This would bring the artifact below the noise floor of the IonCCD. This finding will certainly motivate a new design of the IonCCD allowing it to be mounted even closer to the magnet face experiencing maximum B-fields. In the thesis work of Nishiguchi [34], a different approach of artifact suppression was suggested. Instead of increasing the B', their electro-optical ion detector (EOID) was operated at $\mathrm{B}^{\prime}$ dramatically lowered by using a magnetic shunt at the exit of the magnetic sector, preventing the secondary electrons from returning to the detector. In our case, this approach will not work, as the IonCCD is charge sensitive and would still produce an enhanced signal due to the emitted secondary electrons, leading to overestimation of the $\mathrm{m} / \mathrm{z}$ ion signal. It is exactly this feature, which allows us to experimentally observe the electronic stopping power at play. However, for mass spectrometric reasons especially in isotope ratio measurements such artifacts must be totally suppressed.

We can conclude the following from the above simulations: in cases like this work, where the IonCCD is used directly without an MCP for signal boosting, it should be mounted at the highest $\mathrm{B}$-field possible such that $\mathrm{D}_{2}$ and $\mathrm{D}_{3}$ cancel and $R_{n / p}$ is minimized. The reader should note that the observed artifact is characteristic for potential effect detector like the IonCCD only, since such detectors are sensitive to the charge of the incoming particle. Other pixel detectors such as Medipix/Timepix [28] detectors that are of kinetic effect type rely solely on the impact energy of the particle inducing electron-hole pairs necessary for particle detection. Because of the typically high thresholds $(>4 \mathrm{keV})$, secondary electrons would not be detected as their kinetic energies lie in the sub $10 \mathrm{eV}$ range.

\section{Experiments}

In the following, we present the experimental data needed for comparison to the results predicted by the simulation. We have systematically varied two different ion parameters $(\mathrm{v}, \mathrm{Z})$ and one instrumental parameter $\left(\mathrm{V}_{\mathrm{IonCCD}}\right)$ that influence the secondary electrons. Here, the ion parameters $\mathrm{v}$ and $\mathrm{Z}$ solely influence the secondary electron yield and are thus related to $\gamma$ only. Accordingly, these parameters are expected to influence the artifact integral. As seen in the simulation, $\mathrm{V}_{\text {IonCCD }}$ is expected to influence the dislocation of the returning electrons $\left(d_{e}\right)$ as it accelerates or retards the emitted electrons. Note that by exploiting the rapidly dropping B-field in front of the magnet exit face, $d_{e}$ could also be varied by moving the focal plane away from the magnet. However, we observed that this approach is accompanied by a dramatic change of $\mathrm{W}_{\mathrm{p}}$, especially for low ion mass, which makes the interpretation more complicated.

Figure $4 \mathrm{a}$ displays the experimentally obtained peak distributions for the three detected ions $\mathrm{He}^{+}, \mathrm{N}_{2}{ }^{+}$, and $\mathrm{Ar}^{+}$, obtained at identical impact velocity of 0.05 a.u. $(v(a . u)=.0.2 \times \sqrt{E(k e v / u)})$. The IonCCD was operated at ground potential with no magnet-IonCCD potential gradient, i.e. $\mathrm{V}_{\text {IonCCD }}=0$. The much lower peak intensity observed for $\mathrm{He}^{+}$is mainly due to the low acceleration voltage used $(270 \mathrm{~V})$, which leads to low transmission through the analyzer (see Figure 2). Note that all peaks exhibit the depression at the lower mass side, which is predicted by the simulation. Minimum effect is observed for $\mathrm{He}^{+}$and maximum for $\mathrm{N}_{2}^{+}$. Despite having the largest kinetic energy at equal velocity, $\mathrm{Ar}^{+}$does not exhibit a larger depression than the one observed with $\mathrm{N}_{2}{ }^{+}$. Figure $4 \mathrm{~b}$ shows $\mathrm{R}_{\mathrm{n} / \mathrm{p}}$ values extracted from experimental data for $\mathrm{He}^{+}, \mathrm{N}_{2}{ }^{+}$ and $\mathrm{Ar}^{+}$as a function of the ion velocity. For all ions $\mathrm{R}_{\mathrm{n} / \mathrm{p}}$ is found to increase linearly with ion velocity $v$, a trend that is expected as electronic stopping depends linearly on $v$ (see equation (1). Here, ion maximum velocity is limited by the instruments maximum EI source bias of $2 \mathrm{kV}$. $\mathrm{He}^{+}$exhibits the lowest peak ratio at identical velocity. Being the lightest ion under study, it can be accelerated to highest velocities where maximum $\mathrm{R}_{\mathrm{n} / \mathrm{p}}$ of about $30 \%$ can be obtained. The saturation observed for $\mathrm{He}^{+}$is not due to the saturation of the 

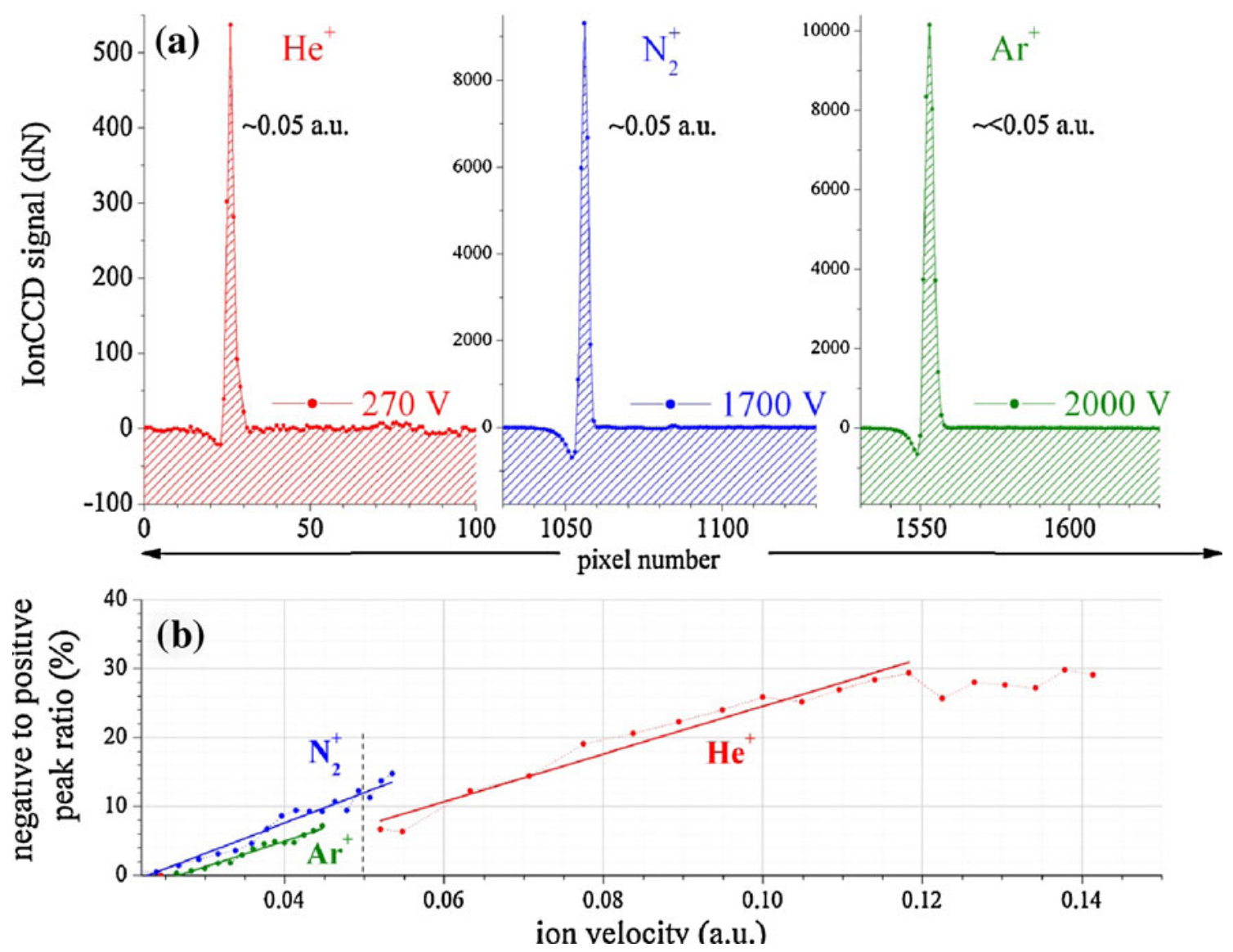

Figure 4. Experimental data showing the stopping power at play. (a) Raw data of different singly charged species detected at a same impact velocity. Note the negative peak on the left side attributed to secondary electron emission. (b) Data analysis showing the negative to positive peak ratio $\left(R_{n / p}\right)$ as function of the ion impact velocity. Note the linearity of this parameter confirming the electronic stopping power process involved

secondary electron yield but to the detector signal saturation, as due to its chip design this version of IonCCD has a limited negative linear dynamic range. In summary it follows from Figure 4 that changes in ion $v$ and $Z$ both markedly influence $R_{n / p}$ as expected for a stopping power related process.

In a second series of experiments we investigated the effect of secondary acceleration deceleration. For this purpose $\mathrm{V}_{\text {IonCCD }}$ was varied over the range from $-100 \mathrm{~V}$ to $+100 \mathrm{~V}$. Electron acceleration towards the magnet exit face is achieved at negative values while positive voltages retard the electron. As an example Figure 5a shows the spectrum of a $1.85 \mathrm{keV} \mathrm{N}_{2}^{+}$ peak for $\mathrm{V}_{\text {IonCCD }}$ values of $-67 \mathrm{~V}, 0 \mathrm{~V}$, and $+67 \mathrm{~V}$. At $0 \mathrm{~V}$ the negative peak is obviously inducing peak distortion, i.e., $R_{n / p}$ is high. Both secondary electron acceleration and deceleration seems to reduce this effect. Figure $5 \mathrm{~b}$ quantifies this result by showing the $\mathrm{R}_{\mathrm{n} / \mathrm{p}}$ as a function of $\mathrm{V}_{\text {IonCCD }}$ in green. Note that for a given electronic stopping process $\left(\mathrm{N}_{2}{ }^{+}\right.$at constant velocity corresponding to $E=1.85 \mathrm{keV}), \mathrm{R}_{\mathrm{n} / \mathrm{p}}$ is drastically altered. A clear maximum is observed around $\mathrm{V}_{\text {IonCCD }}=-5 \mathrm{~V}$. For stronger acceleration, $\mathrm{R}_{\mathrm{n} / \mathrm{p}}$ swiftly decreases to zero. This phenomenon is probably due to the increase of secondary electron displacement $\left(\mathrm{d}_{\mathrm{e}}\right)$ to a level, where the electrons return outside the active detector area. Conversely, retarding the electrons $\left(\mathrm{V}_{\text {IonCCD }}>0\right)$ leads to a decrease of $\mathrm{R}_{\mathrm{n} / \mathrm{p}}$, but not to zero. In this case, the negative peak at the left side decreases to a certain level while a second negative peak appears on the higher mass side of the major peak (see gray line peak in Figure 5a). The associated plateau observed in Figure 5b (green curve) is the contribution of the two smaller peaks. The appearance of the second, low signal, negative peak is not yet understood. Discrepancies between simulation and experiment could be due to the insulator gaps between the TiN pixels, inhomogeneity and surface roughness of the pixels, contamination layers (discussed in the next section), and the curvature of the B-field assumed to be transversal in the model or the focused ion beam distribution on the detector. All these issues are not accounted for in the simulation. From Figure $5 \mathrm{~b}$ it is furthermore obvious, that any bias on the IonCCD slightly degrades the instrument FWHM resolving power (blue curve) from its optimum resolution $\mathrm{V}_{\text {IonCCD }}=0 \mathrm{~V}$ if no compensating fields are applied in the MH-mass spectrometer optics. This slight deterioration of the resolving power is caused by the Efield disturbing the field-free region, which is a focusing condition of the MH-mass spectrometer. To conclude, 

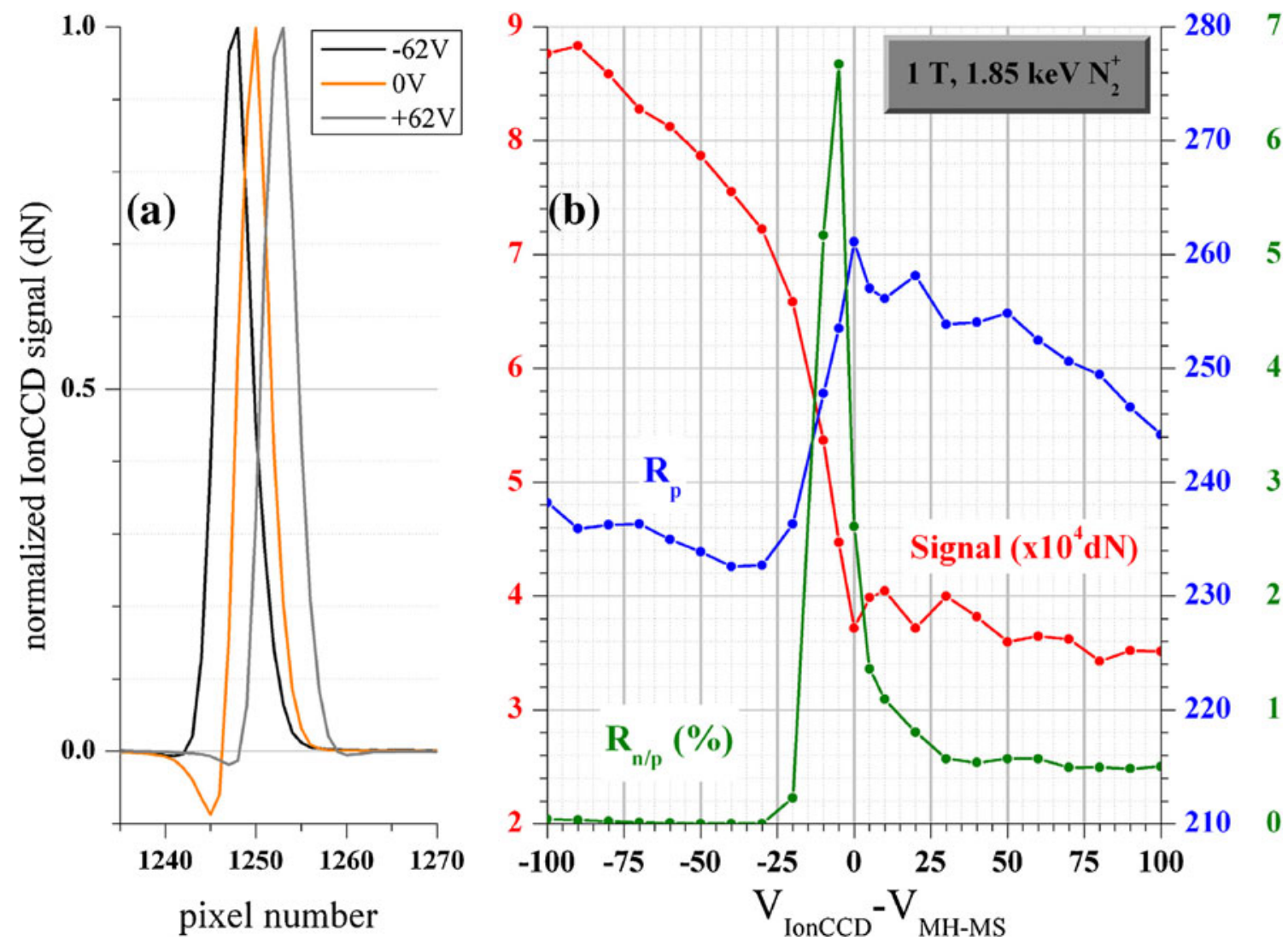

Figure 5. Experimental results showing the retarding field. (a) Peak shape of the $1.85 \mathrm{keV} \mathrm{N}_{2}^{+}$peak at three lonCCD biases. (b) Summary of the experimental results plotted as function of the potential gradient in the interface magnet exit face-lonCCD. The red curve is the peak area of the detected $\mathrm{N}_{2}{ }^{+}$ion. The blue curve is the extracted resolving power at $50 \%$ peak height of the instrument. The green curve is the negative to positive peak ratio $\left(R_{n / p}\right)$ directly related to the electronic stopping power

modification of retarding field is not a suitable method for artifact suppression.

The 2-fold increase in the signal shown in Figure $5 b$ (red curve) at negative IonCCD bias is unexpected. This increase is beyond the focusing effect of the biased IonCCD and calls for further investigation.

A very exciting unknown feature of the IonCCD could be discovered by systematic variation of $\mathrm{V}_{\text {IonCCD. }}$. Upon increase of $\mathrm{V}_{\text {IonCCD }}$ from $0 \mathrm{~V}$ to $+1100 \mathrm{~V}$, the increase in deceleration of the $45^{\circ}$ incident $1.85 \mathrm{keV}$ ions first lead to a signal decrease accompanied by a peak shift in the high mass direction. Eventually, the signal is lost and a negative signal appears which is dislocated from the initial peak position. The negative signal exhibits a double structure for all ions under study. This structure can be reproduced in the simulation, using velocity reversal analysis in the IonCCD region under the assumption that image charges induced by the deflected ion in the IonCCD material are causing the effect. This discovery is very interesting, since it broadens the range of applications for the IonCCD from incoming negative ions and electrons (as demonstrated previously [2]) to ion glancing induced image charge formation in the detector surface. A detailed experimental and simulative study of this effect is beyond the scope of this article and will be subject to a subsequent work.

\section{Surface Characterization}

For the experiments reported here and a in a previous study [2], we exposed the TiN surface of the IonCCD electrodes to several types of projectiles, ranging from $250 \mathrm{eV}$ electrons over hyperthermal biomolecules $(<20 \mathrm{eV},>1100 \mathrm{u}$, obtained from an ESI source) up to $\mathrm{keV}$ ions $(2 \mathrm{keV}, 4-40 \mathrm{u}$, obtained from an EI source). At relatively low electron energies $(<250 \mathrm{eV})$; electrons have virtually no chemical or physical effect on the electrodes due to the very low momentum involved. As demonstrated in the previous work [2], detection of low energy and high molecular ion mass shows no effect on the inert TiN surface either. In the low eV/u regime $(<0.1 \mathrm{eV} / \mathrm{u})$, soft-landing of the species is the underlying process and no damage is inflicted on the surface, as well. We showed earlier that soft-landing of biomolecules on the detector only result in a gentle deposition of the detected material on the surface. The induced discoloration of the exposed pixels can easily be rinsed off using a methanol wetted cotton swab. This clearly 
shows that the deposited material was not covalently bound to the chemically inert TiN surface. However at $\mathrm{keV}$ energies employed here and ideally suited for focal plane sector field (MH-mass spectrometer) and distance of flight (DOF) $[60,61]$ instrumentation, a relatively strong effect of ion impact on the detector surface is observed.

It is well established that interaction of $\mathrm{keV}$ primary ions with the detector surface typically leads to a penetration depth between a few $\mathrm{nm}$ and a couple of $10 \mathrm{~nm}$. It has three major effects: (1) mixing of the upper layers of the sample, resulting in an amorphization of the surface; (2) implantation of atoms from the primary ion beam into the sample, and (3) ejection of secondary particles (atoms and small molecules), i.e., sputtering. The latter effect is exploited in the powerful surface characterization technique SIMS [7-9].

For surface analytical investigation, a chip that was extensively used on-and-off for a period of 1 year in an MH-mass spectrometer instrument was used. Shown in Figure $6 \mathrm{a}$ is a photograph of the pixels around the impact area where $1 \mathrm{keV} \mathrm{N}_{2}{ }^{+}$ions were detected. In this case, the discoloration of the exposed pixels could not be rinsed off, as was possible for soft-landed ions [2]. The discoloration of the exposed pixels is the result of $1 \mathrm{keV}$ ion impact induced permanent surface modification. However, no signs of sensitivity loss were observed when using the IonCCD for $\mathrm{keV}$ ion detection. Due to the charge sensitivity of the detector, only electrical conduction properties of the surface would induce charge sensitivity losses. As long as no predominant change is the conduction occurs no sensitivity loss should be expected. An experimental verification can be found in our earlier work [2], where we show that multilayer soft-landing did not degrade the IonCCD sensitivity to further soft-landed protonated peptides. The IonCCD detection efficiency, as far as pixel surface modification is concerned, is as stable as the electrical conduction property of the pixel surface remains constant.

We have then studied the morphology of unexposed and exposed areas of the TiN pixel surface using Atomic Force

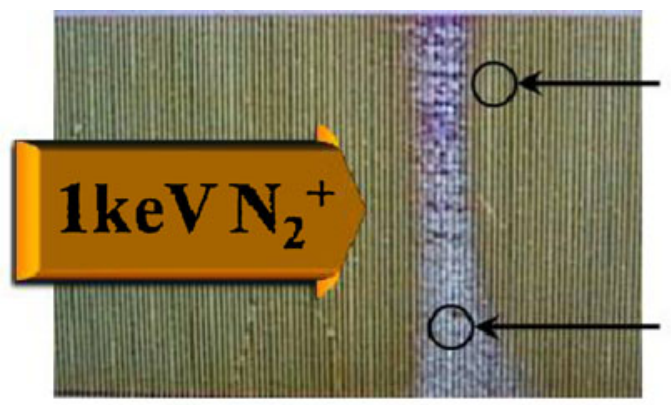

(a)

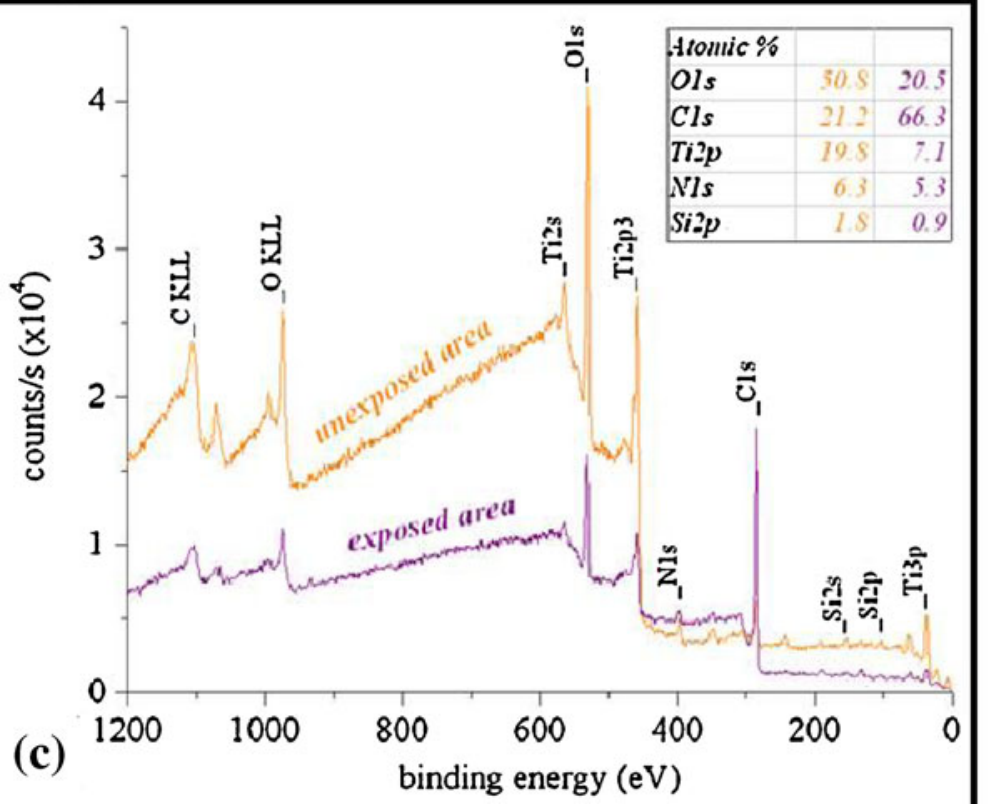

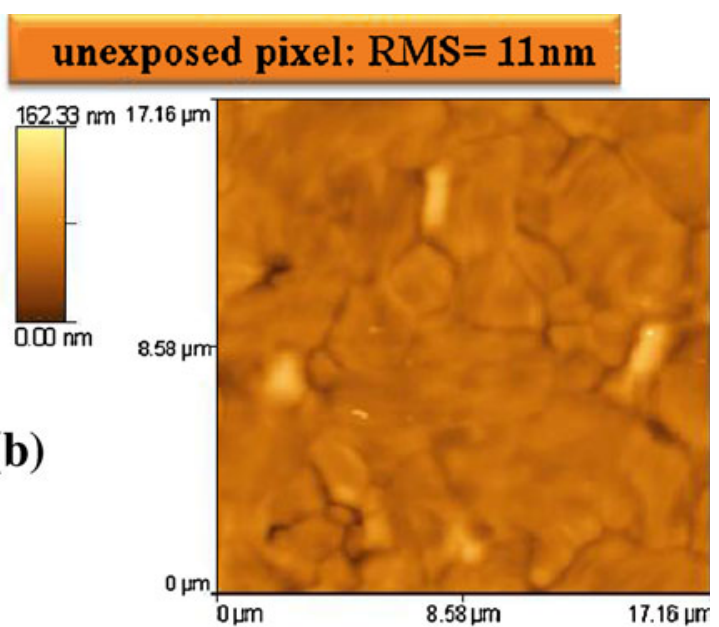

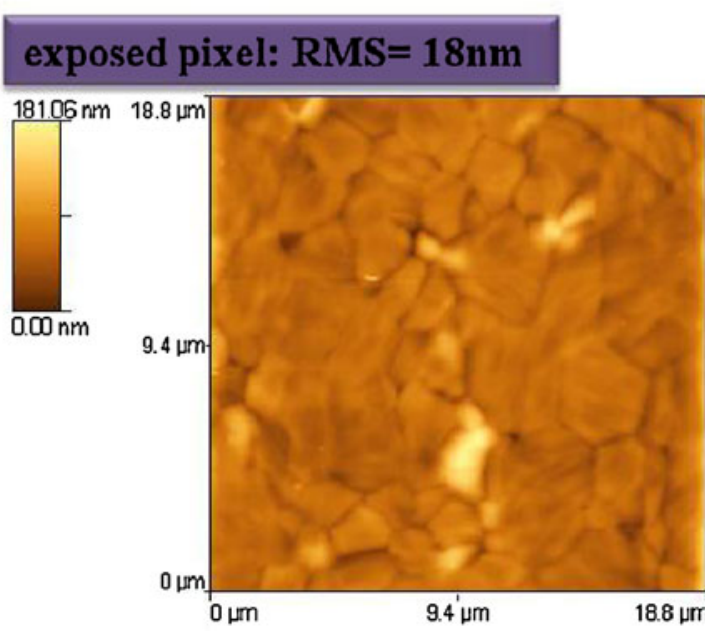

Figure 6. IonCCD surface characterization. (a) Photograph of the $1 \mathrm{keV} \mathrm{N}_{2}^{+}$ion impact region on the array detector. (b) Contact mode Atomic Force Microscope images of the unexposed pixel (top) and exposed pixel (bottom). (c) XPS spectrum of the unexposed and exposed regions in orange and purple respectively 
Microscopy measurements in Contact Mode (AFM-CM). The acquired images displayed in Figure 6b show a roughness increase (from 11 to $18 \mathrm{~nm}$ RMS) comparing exposed with unexposed pixel areas. This phenomenon is in agreement with the general trend of surface roughening upon $\mathrm{keV}$ ion impact [62]. As for the observed discoloration from gold-like color for unexposed TiN to a purple-like color for exposed TiN, the measured roughness increase might play a role. Another possible explanation for the discoloration is preferential sputtering of nitrogen [63] changing the stoichiometry of the alloy, or ion implantation inducing a similar effect.

In order to investigate the discoloration in more detail, we employed X-ray photoelectron-spectroscopy (XPS). The results are shown in Figure 6c comparing again exposed to unexposed pixel areas. XPS spectra obtained from the raw surfaces were contaminated with carbon and oxygen due to ambient air storage of the detector. The displayed spectra, however, where taken after removal of the contaminating layers by argon ion sputtering of the topmost $10 \AA$ from the surface (Figure 6c).

In the spectra, strong N1s and Ti2p3 peaks at 396.5 and $458 \mathrm{eV}$ are due to the pristine detector coating material. The $\mathrm{O} 1 \mathrm{~s}$ at $530.5 \mathrm{eV}$ is due to oxide formation on the surface [64]. The C1s peak at $284.5 \mathrm{eV}$ is due to hydrocarbon contamination [65-68]. Comparison of spectra for the unexposed and exposed surfaces reveals an overall decrease in photoelectron yield. On a relative scale, the most obvious change is the increase of the C1s peak, which is dominant for the exposed surface. Note that this dominance could not be removed by moderate sputtering. The $\mathrm{keV}$ ion-induced roughening of the surface apparently increases the hydrocarbon-sticking coefficient of the detector surface dramatically. Surprisingly, we observe no indication for nitrogen and xenon (when probing the xenon beam impact area) implantation or for preferential sputtering.

\section{Conclusion}

In this article, we presented results from a 3D ion trajectory simulation of our miniature sector field instrument of Mattauch-Herzog geometry to clarify the origin of the artifact manifesting in the mass spectra as negative extra peaks. By comparison with a systematic experimental study of this artifact, it could be related to electronic stopping. We demonstrated that the artifact increases linearly with ion impact velocity and is dependent, in an oscillatory fashion, on ion nuclear charge. Both findings are in agreement expectations from electronic stopping of $\mathrm{keV}$ ions within the TiN surface layer of the IonCCD, leading to electronic excitation and secondary electron emission. The 3D simulation showed that operating the IonCCD detection surface at higher B-field $(>4000 \mathrm{G})$ can efficiently eliminate the peak artifact. Future IonCCD designs will thus have to allow the chip surface to be as close as possible to the magnet. A $\mathrm{kV}$ floating bias of the IonCCD and the camera system confirmed the causal relationship between the observed artifact and secondary electron emission but was found unsuitable for artifact reduction.

Excitingly, we observed sensitivity of the IonCCD detector to image charge formation induced by glancing ions for the first time. This newly discovered capability will be the focus of future work as it could open new horizons for the IonCCD as a position-sensitive image charge integrator in nondestructive measurements.

In order to investigate potential IonCCD damage upon $\mathrm{keV}$ ion impact through the nuclear stopping effect, AFM and XPS surface characterization techniques were used. While AFM confirmed the expected increase in surface roughness, XPS showed no signature of either ion implantation or preferential ion sputtering after extensive use of the IonCCD. The discoloration observed after extensive use was found to be due to carbon layer formation in the roughened, irradiated pixel area. However, as long as nuclear stopping effects do not change the conductive properties of the pixel electrodes and the gaps, the detector performance should not be affected.

\section{Acknowledgments}

The authors acknowledge support for this work by OI Analytical and University of North Texas for XPS surface characterization and the University of Alabama at Birmingham for AFM imaging. O.H. acknowledges the support of OI Analytical for this research. O.H. acknowledges Dr. Theva Thevuthasan for the enlightening surface science discussions. The work was performed at the CMS Field Products subsidiary of OI Analytical and at the University of North Texas and the University of Alabama at Birmingham.

\section{References}

1. Sinha, M., Wadsworth, M.: Miniature focal plane mass spectrometer with 1000-pixel modified-CCD detector array for direct ion measurement. Rev. Sci. Instrum. 76(2) (2005)

2. Hadjar, O.: IonCCD ${ }^{\mathrm{TM}}$ for direct position-sensitive charged-particle detection: from electrons and $\mathrm{keV}$ ions to hyperthermal biomolecular ions. J. Am. Soc. Mass Spectrom. 22(4), 612-624 (2011)

3. Sinha, M., Tomassian, A.D.: Development of a miniturized, lighweight magnetic-sector for a field-portable mass spectograph. Rev. Sci. Instrum. 62(11), 2618-2620 (1991)

4. Burgoyne, T.W., Hieftje, G.M., Hites, R.A.: Design and performance of a plasma-source mass spectrograph. J. Am. Soc. Mass Spectrom. 8(4), 307-318 (1997)

5. Nishiguchi, M.: Ion optical evaluation of a miniature double-focusing mass spectrograph. Eur. J. Mass Spectrom. 14(1), 7-15 (2008)

6. Benninghoven, A.: Chemical analysis of incorganic and organic surfaces and thin films by static time-of-flight secondasry ion mass spectrometry (TOF-SIMS). Angew. Chem. Int. Ed. 33(10), 1023-1043 (1994)

7. Van Vaeck, L., Adriaens, A., Gijbels, R.: Static secondary ion mass spectrometry: (S-SIMS) Part 1. Methodology and structural interpretation. Mass Spectrom. Rev. 18(1), 1-47 (1999)

8. Adriaens, A., Van Vaeck, L., Adams, F.: Static secondary ion mass spectrometry (S-SIMS) Part 2. Material science applications. Mass Spectrom. Rev. 18(1), 48-81 (1999)

9. Nojima, A.: Nanoscale SIMS analysis: the next generation in local analysis. Appl. Surf. Sci. 203, 194-197 (2003) 
10. Opila, R.L., Eng, J.: Thin films and interfaces in microelectronics: composition and chemistry as function of depth. Prog. Surf. Sci. 69(4/6), $125-163(2002)$

11. Gallon, T.E., Matthew, J.A.D.: Auger electron spectroscopy and its application to surface studies. Rev. Phys. Technol. 3(1), 31-64 (1972)

12. Gallon, T.E., Orgassa, K., Matthew, J.A.D.: Low energy auger electron emission from titanium induced by ion bombardment. J. Phys. Condens. Matter 7(45), 8539-8546 (1995)

13. Broers, A.N.: Fabrication limits of electron beam lithography and of UV, X-ray, and ion-beam lithographies. Philos. Trans. Royal Soc. London Ser A-Math. Phys. Eng. Sci. 353(1703), 291-311 (1995)

14. Broers, A.N., Hoole, A.C.F., Ryan, J.M.: Electron beam lithographyResolution limits. Microelectron. Eng. 32(1/4), 131-142 (1996)

15. Reyntjens, S., Puers, R.: A review of focused ion beam applications in microsystem technology. J. Micromech. Microeng. 11(4), 287-300 (2001)

16. Momota, S.: Ion-beam lithography by use of highly charged Ar-ion beam. Rev. Sci. Instrum. 77(3) (2006)

17. Fraser, G.W.: The ion detection efficiency of microchannel plates (MCPs). Int. J. Mass Spectrom. 215(1/3), 13-30 (2002)

18. Hedin, A., Hansson, Sundqvist, B.U.R.: On the detection of large organic ions by secondary electron production. Int. J. Mass Spectrom. Ion Processes 75, 275-298 (1987)

19. Le Beyec, Y.: Cluster impacts at $\mathrm{keV}$ and $\mathrm{MeV}$ energies: Secondary emission phenomena. Int. J. Mass Spectrom. Ion Processes 174, 101-117 (1998)

20. Baragiola, R.A.: Electron emission from surfaces by impact of polyatomic ions and cosmic dust. Nucl. Instrum. Methods Phys. Res. $B$ 88, 35-43 (1994)

21. Winter, H.: Suppression of potential electron emission for impact of slow multicharged fullerenes on clean gold. Phys. Rev. A 56, $3007-$ 3010 (1997)

22. Comisarow, M.B., Marshall, A.G.: Fourier transform ion cyclotron resonance spectroscopy. Chem. Phys. Lett. 25(282/283) (1974)

23. Marshall, A.G., Hendrickson, C.L., Jackson, G.S.: Fourier transform ion cyclotron resonance mass spectrometry: A primer. Mass Spectrom. Rev. 17(1), 1-35 (1998)

24. Marshall, A.G., Hendrickson, C.L.: Fourier transform ion cyclotron resonance detection: Principles and experimental configurations. Int. J. Mass Spectrom. 215(1/3), 59-75 (2002)

25. Makarov, A.: Electrostatic axially harmonic orbital trapping: A highperformance technique of mass analysis. Anal. Chem. 72(6), 1156-1162 (2000)

26. Hu, Q.Z.: The Orbitrap: A new mass spectrometer. J. Mass Spectrom. 40(4), 430-443 (2005)

27. Llopart, X.: Medipix2: a 64-k pixel readout chip with $55 \mu \mathrm{m}$ square elements working in single photon counting mode. IEEE Trans. Nucl. Sci. 49(5), 2279-2283 (2002)

28. Gademann, G.: Velocity map imaging using an in-vacuum pixel detector. Rev. Sci. Instrum. 80(10), 103105 (2009)

29. Jungmann, J.: Fast High Resolution Mass Spectrometry Imaging Using a Medipix Pixelated Detector. J. Am. Soc. Mass Spectrom. 21(12), 2023-2030 (2010)

30. Mazza, G.: The NA62 Gigatracker pixel detector system. Nucl. Instrum. Methods Phys. Res. Sect. A Acceler. Spectrom. Detect. Assoc. Equip. 617(13), 558-559 (2010)

31. Nomerotski, A.: Pixel Imaging Mass Spectrometry with fast and intelligent Pixel detectors. J. Instrum. 5 (2010)

32. Schilling, G.D.: Characterization of a second-generation focal-plane camera coupled to an inductively coupled plasma Mattauch-Herzog geometry mass spectrograph. Anal Chem 78(13), 4319-4325 (2006)

33. Johnson, E., Hadjar, O., Laskin, J.: Characterization of the Ion Beam Focusing in a Mass Spectrometer Using an IonCCD. J. Am. Soc. Mass Spectrom. (2011), accessed online, 18 May.

34. Nishiguchi, M.: Development of miniature double-focusing mass spectrograph for lunar exploration. In: Department of Physics, Graduate School of Science, p. 110. Osaka University, Osaka (2006)

35. Binnig, G., Quate, C.F.: Atomic Force Microscope. Phys. Rev. Lett. 56, 930-933 (1986)

36. Rutherford, E.: Philos Mag B Phys Condens Matt Stat Mech Electron Opt Magn Prop 21, 669 (1911)

37. Bohr, N.: Philos Mag B-Phys Conden Matt Stat Mech Electron Opt Mag Prop 25, 16 (1913)
38. Bethe, H.A.: Zur Theorie des Durchgangs schneller Korpuskularstrahlen durch Materie. Ann. Phys. (Leipzig) 5, 325 (1930)

39. Arnau, A.: A charge state approach to the stopping power of ions in solids. Nucl. Instrum. Methods Phys. Res. B Beam Interact Materials Atoms 69(1), 102-107 (1992)

40. Echenique, M.: Non Linear Stopping Power of an Electron Gas for Slow Ions. Phys Rev A 33, 897 (1986)

41. Echenique, M., Nieminen, R.M., Ritchie, R.H.: Density Functional Calculation of Stopping Power of an Electron Gas for Slow Ions. Solid State Commun. 37, 779 (1981)

42. Penalba, M.: Stopping power for protons in aluminum. Europhys. Lett. 19(1), 45-50 (1992)

43. Ferrel, T.L., Ritchie, R.H.: Energy losses by slow ions and atoms to electronic excitation in solids. Phys. Rev. B 16, 115 (1977)

44. Puska, M.J., Nieminen, R.M.: Atoms embedded in an electron gas: Phase shifts and cross sections. Phys. Rev. B 27, 6121 (1983)

45. Ferrell, T.L., Echenique, M., Ritchie, R.H.: Friction parameter of an ion near a metal surface. Solid State Commun 32(5), 419-422 (1979)

46. Abril, I., Garciamolina, R., Arista, N.R.: Proton energy loss in allotropic forms of carbon. Nucl Instrum Methods Phys Res Sect B Beam Interact Materials Atoms 90(14), 72-75 (1994)

47. Child, M.S.: Molecular Collision Theory. Dover Publications (1996)

48. Schlatholter, T.: Electronic stopping in ion-fullerene collisions. Appl. Phys. A-Materials Sci. Processing 72(3), 281-287 (2001)

49. Schlatholter, T.: Strong velocity effects in collisions of $\mathrm{He}+$ with fullerenes. Phys. Rev. Lett. 82(1), 73-76 (1999)

50. Rothard, H., Schou, J., Groeneveld, K.O.: Projectile-state-dependent and charge-state-dependent elecron yields from ion penetration of solids as a probe of pre-equilibrium stopping power. Phys. Rev. A 45(3), 1701-1710 (1992)

51. Wang, Y.N., Ma, T.C., Gong, Y.: Z1 oscilation in electronic stopping power for slow ions. Phys. Lett. A 167(3), 287-290 (1992)

52. Hadjar, O.: Z oscillations in ion-induced fullerene fragmentation. Phys. Rev. Lett. 84(18), 4076-4079 (2000)

53. Hadjar, O.: Projectile atomic-number effect on ion-induced fragmentation and ionization of fullerenes. Phys. Rev. A 63(3) (2001)

54. Aumayr, F., Winter, H.: Inelastic interactions of slow ions and atoms with surfaces. Nucl. Instrum. Methods Phys. Res. Sect. B Beam Interact. Materials Atoms 233, 111-124 (2005)

55. Schlatholter, T., Hoekstra, R., Morgenstern, R.: Collisions of Oq + with neutral C-60: Charge transfer and fragmentation. J. Phys. B-Atomic Mol. Optical Phys 31(6), 1321-1331 (1998)

56. Eder, H.: Projectile charge effects in ion-induced kinetic electron emission from solid surfaces. Physica Scripta T80B, 236-237 (1999)

57. Baragiola, R.A.: Principles and mechanisms of ion induced electron emission. Nucl. Instrum. Methods in Phys. Res. B 78, 223-238 (1993)

58. Mattauch, J., Herzog, R.: Über einen neuen Massenspektrographen. Z. Phys. 89 (1934)

59. Suharyanto: Secondary electron emission of TiN-coated alumina ceramics. Vacuum 81(6), 799-802 (2007)

60. Enke, C.G., Dobson, G.S.: Achievement of energy focus for distanceof-flight mass spectrometry with constant momentum acceleration and an ion mirror. Anal. Chem. 79, 8650-8661 (2007)

61. Graham, A.W.G.: First Distance-of-Flight Instrument: Opening a New Paradigm in Mass Spectrometry. J. Am. Soc. Mass Spectrom 22(1), 110-117 (2011)

62. Smentkowski, V.S.: Trends in sputtering. Prog. Surf. Sci. 64(1/2), 1-58 (2000)

63. Ranjan, R.: Absolute sputtering yield of Ti/TiN by $\mathrm{Ar}+\mathrm{N}+$ at $400-700 \mathrm{eV}$. J Vacuum Sci. Technol. A 19(3), 1004-1007 (2001)

64. Huang, M.: XPS observation of surface interaction between $\mathrm{H}-2$ and $\mathrm{CO}_{2}$ on platinum foil. J Mol Catalysis A Chem. 104(2), L131-L137 (1995)

65. Veziroglu, T.N., Zginaichenko, S.Y., Schur, D.V.: NATO Security through Science Series A: Chemistry and Biology. In: Veziroglu, T.N., Zginaichenko, S.Y., Schur, D.V. (eds.) Hydrogen Materials Science and Chemistry of Carbon Nanomaterials. Springer, Dortrecht (2007)

66. Yakshinskiy, B.V.: Electron-induced interaction of selected hydrocarbons with $\mathrm{TiO} 2$ surfaces: The relevance to extreme ultraviolet lithography. J. Phys. Condensed Matter 22(8), (2010)

67. Hollenshead, J., Klebanoff, L.: Modeling radiation-induced carbon contamination of extreme ultraviolet optics. J. Vacuum Sci. Technol. B 24(1), 64-82 (2006)

68. Klebanoff, L.E.: Radiation-induced protective carbon coating for extreme ultraviolet optics. J. Vacuum Sci. Technol. B 20(2), 696-703 (2002) 